\title{
Proposta e seleção de uma função polinomial para a avaliação de sistemas de irrigação
}

\author{
Euzebio M. da Silva ${ }^{1}$, Jorge E. F. W. Lima ${ }^{1} \&$ Juscelino A. de Azevedo ${ }^{1}$
}

\begin{abstract}
RESUMO
O uso de modelos matemáticos facilita o cálculo dos parâmetros de uniformidade e eficiência de sistemas de irrigação e, por isso, sua utilização deve ser incentivada. Com este trabalho se propõe o uso de uma função polinomial na avaliação de sistemas de irrigação e desenvolver as relações matemáticas para o cálculo dos principais indicadores de desempenho, utilizando-se esta função. Para definir o modelo a ser proposto e verificar sua aplicabilidade, realizaram-se comparações entre os resultados obtidos com funções polinomiais de vários graus e o modelo Potencial Silva, utilizando-se dados de 91 casos de avaliação de desempenho de diversos sistemas de irrigação. A seleção do modelo polinomial mais adequado foi efetuada por meio da comparação de curvas ajustadas à distribuição das freqüências acumuladas da soma de quadrados dos erros, obtidos no ajuste dos modelos a cada conjunto de valores de água aplicada. Os resultados revelaram que a função polinomial do quinto grau é a recomendada para descrever perfis de distribuição da água aplicada por sistemas de irrigação e derivar expressões matemáticas para o cálculo dos indicadores de desempenho correspondentes.
\end{abstract}

Palavras-chave: água aplicada, uniformidade, eficiência, distribuição de água

\section{Proposal and selection of a polynomial function for evaluating irrigation systems}

\begin{abstract}
The use of mathematical models facilitates the calculation of the uniformity and efficiency parameters of irrigation systems and, thus, its utilization should be encouraged. This work had as its objectives the proposal of the use of a polynomial function for evaluating irrigation systems and to develop the mathematical relationships for calculating the main indicators for measuring irrigation performance, utilizing this function. In order to define the model to be proposed and to verify its suitability, comparisons were carried out among polynomial functions of various degrees against the Silva's potential model, utilizing data from 91 cases of irrigation system evaluations. The selection of the most adequate polynomial model was made by comparing adjusted curves to the cumulative frequency distribution of the sum of square errors that were obtained after adjusting the models to each set of measured water application data. The results showed that the fifth degree polynomial function is recommended to describe the applied water distribution from irrigation systems and it can be used to derive mathematical expressions for calculating the corresponding performance measures.
\end{abstract}

Key words: applied water, uniformity, efficiency, water distribution 


\section{INTRODUÇÃO}

O desenvolvimento de modelos matemáticos para o ajuste e avaliação da distribuição espacial da água aplicada, apesar de ser uma alternativa importante nos estudos acerca do desempenho de sistemas da irrigação, ficou um longo período ausente de textos científicos e apenas recentemente voltou a ser objeto de publicações, com a proposição de novos modelos. Dentre os avanços recentes se destaca a proposição de um modelo não-linear para a análise de desempenho da irrigação (Silva et al., 2004), que apresentou resultados de ajuste aos dados de avaliação de desempenho da irrigação estatisticamente superiores aos dos principais modelos não-lineares normalmente utilizados (Silva et al., 2006). Uma das principais características dos modelos não-lineares utilizados, exceto o de Karmeli (1978) e o de Silva et al. (2004), é o fato de se basearem em funções estatísticas de probabilidade de distribuição: Normal (Hart \& Reynolds, 1965), Gama (Chaudry, 1978) e Beta (Elliot et al., 1980).

Antes da popularização dos computadores, a opção pelos modelos probabilísticos era naturalmente justificada pela disponibilidade de tabelas estatísticas que possibilitavam a consulta de valores de probabilidade acumulada, os quais eram tomados como numericamente equivalentes à fração da área total irrigada, calculada com base na área representada em cada ponto de coleta da água aplicada pelo sistema de irrigação. Com a evolução dos recursos computacionais e o acesso à informática cada vez mais facilitado, tornou-se viável o uso de outros tipos de modelos que não fossem necessariamente estatísticos.

Naturalmente, uma vez superada esta limitação, o critério de escolha do modelo deve ser centrado em sua flexibilidade de ajuste aos mais variados tipos de distribuição da água aplicada pelos diversos sistemas de irrigação; além disso, o modelo adotado deve permitir com facilidade a derivação dos indicadores de uniformidade e de eficiência da irrigação e sua incorporação em programas computacionais destinados à avaliação de desempenho e manejo da água de sistemas de irrigação. Com essas justificativas, considerou-se pertinente a idéia de continuar a busca por novos modelos matemáticos e que não sejam necessariamente baseados em funções estatísticas de probabilidade mas que possam reproduzir, o mais fielmente possível, a maioria dos formatos da distribuição das lâminas de água aplicada por sistemas de irrigação.

Dentre as possibilidades de modelos ainda não testados para a avaliação de desempenho de sistemas de irrigação se destacam os de natureza polinomial, os quais, em decorrência de sua linearidade, podem, em princípio, favorecer a derivação das expressões matemáticas explícitas para o cálculo dos indicadores de uniformidade e de eficiência da irrigação. Uma vez comprovada a viabilidade dessa proposição, um dos primeiros passos a ser considerado para sua aplicação será a escolha da função polinomial de menor grau que resulte no melhor ajuste possível, tomando-se por base um conjunto representativo de dados de distribuição da água aplicada de diversos sistemas de irrigação.

Com este trabalho objetivou-se propor o uso de uma função polinomial na avaliação de sistemas de irrigação e de- senvolver as relações matemáticas para o cálculo dos principais indicadores de desempenho da irrigação, utilizandose tal função.

\section{MATERIAL E MÉTODOS}

Com o mesmo conjunto de dados de Silva et al. (2006), proveniente de 91 casos de avaliação de desempenho de sistemas de irrigação, testaram-se as funções polinomiais do terceiro ao décimo graus, ajustando-as aos respectivos perfis de distribuição de água aplicada representados na forma de lâminas de água decrescentes em função da fração da área total irrigada. Nesse ajuste, as lâminas de água aplicada representaram a variável dependente do modelo e a fração da área total irrigada, a variável independente. Os valores dos coeficientes de cada função polinomial foram obtidos aplicando-se o método dos mínimos quadrados (Souza, 1998). Na seleção dos perfis de distribuição para este trabalho tevese o cuidado de considerar somente os casos de avaliação de desempenho em que toda a área irrigada recebeu, efetivamente, alguma aplicação de água, ou seja, não houve coleta nula de água, a fim de evitar situações em que as funções polinomiais, principalmente as de maiores graus, predissessem valores negativos de lâminas de água aplicada, o que não seria coerente.

Para selecionar, dentre as funções polinomiais testadas, aquela de menor grau que melhor representasse os perfis de distribuição de água analisados, tomou-se para comparação o modelo Potencial Silva (Silva et al., 2004), que foi demonstrado (Silva et al., 2006) possuir o melhor desempenho entre os modelos não-lineares mais utilizados. Para construir a ferramenta estatística de diferenciação entre os modelos e selecionar o grau da função polinomial que apresentasse um desempenho de ajuste superior ao modelo Potencial Silva, utilizaram-se os erros correspondentes à soma de quadrados das diferenças entre os valores observados e os calculados, provenientes da aplicação de cada modelo testado no ajuste aos 91 casos da avaliação de desempenho utilizados (Silva et al., 2006). O valor de cada erro foi dividido pela diferença entre o número de observações e o respectivo número de parâmetros do modelo (Draper \& Smith, 1998) resultando, assim, na variável SQE (Soma do Quadrado dos Erros), a qual serviu de base para a diferenciação entre os modelos testados.

Para gerar a função de probabilidade usada na comparação estatística entre os modelos considerados, o conjunto dos valores de SQE correspondente a cada modelo foi analisado na forma de histograma de freqüências, agrupados em sete classes, e ajustado à função exponencial de probabilidade acumulada, seguindo-se os mesmos procedimentos de análises utilizados em Lima et al. (2006) e Silva et al. (2006). As funções de probabilidade resultantes foram comparadas, duas a duas, utilizando-se o teste estatístico apresentado em Silva \& Azevedo (2002), reescrito da seguinte maneira:

$$
\mathrm{F}=\frac{\left|\mathrm{SQE}_{\text {combinaç̃o }}-\left(\mathrm{SQE}_{\text {Potencial Silva }}+\mathrm{SQE}_{\text {Polinomial }}\right)\right| / \mathrm{GL}_{\text {num }}}{\left(\mathrm{SQE}_{\text {Potencial Silva }}+\mathrm{SQE}_{\text {Polinomial }}\right) / \mathrm{GL}_{\text {den }}}
$$


sendo F definido como o valor da razão entre o resultado das variâncias do numerador (num) e do denominador (den); $\mathrm{SQE}_{\text {Potencial Silva }}$ e $\mathrm{SQE}_{\text {Polinomial, a soma de quadrados dos re- }}$ síduos, resultantes do ajuste da função de probabilidade exponencial às respectivas freqüências acumuladas dos valo-

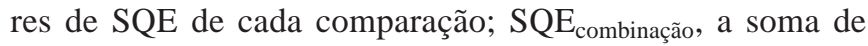
quadrados dos resíduos resultantes do ajuste da função probabilística ao conjunto das freqüências acumuladas dos dois modelos comparados; $\mathrm{GL}_{\text {num }}$ e $\mathrm{GL}_{\mathrm{den}}$, os graus de liberdade do numerador e do denominador, considerando-se as sete classes de SQE e os dois parâmetros da função de probabilidade exponencial.

Após ter sido determinada a função polinomial de menor grau com capacidade de ajuste aos dados observados superior ao modelo Potencial Silva (Silva et al., 2004), utilizando-se os 91 casos de avaliação de desempenho de sistemas de irrigação (Silva et al., 2006), então o passo seguinte consistiu em desenvolver as expressões matemáticas que deveriam ser empregadas no cálculo dos indicadores de desempenho da irrigação. É importante destacar que nessa modelagem não foram incluídas as perdas e desuniformidades devidas ao escoamento superficial na área objeto da aplicação de água.

\section{RESULTADOS E DISCUSSÃO}

Analisando-se os histogramas das freqüências dos valores de SQE médio resultantes do ajuste das funções polinomiais do terceiro ao décimo grau em conjunto com o modelo Potencial Silva (Silva et al., 2004), constatou-se que a função estatística exponencial poderia ser empregada para representar adequadamente a distribuição das probabilidades acumuladas desses resíduos (Figura 1) e, assim, ser utilizada também como ferramenta de discriminação entre os modelos e quantificação estatística das diferenças entre eles.

Com base nesta análise, foi possível verificar que a função polinomial do quinto grau foi suficiente para superar o modelo Potencial Silva dispensando, assim, da análise, as polinomiais de maior grau. Verificou-se, também, estreita concordância entre a função de probabilidade exponencial e as frequências acumuladas dos valores de SQE, empregando-se o teste de significância $r$ de Pearson nas correlações obtidas entre esses valores e os calculados com o modelo de distribuição exponencial. Nesses ajustes foram observadas as seguintes correlações: Potencial Silva $(r=0,97)$; Polinomial do terceiro grau $(r=0,99)$; Polinomial do quarto grau $(r=0,99)$; Polinomial do quinto grau $(r=0,99)$, todas com um nível de significância de quase $100 \%$.

Com esse esquema de representação (Figura 1), quanto mais próxima do eixo das probabilidades estiver a curva traçada, menores serão, relativamente, os valores de SQE e, consequentemente, melhor o modelo. Confrontando a média dos valores de SQE do modelo Potencial Silva (Silva et al., 2004), tomado como referência, com as médias dos valores de SQE das respectivas funções polinomiais testadas, verificou-se que a função polinomial do quinto grau, com um SQE médio de 0,00095 , foi superior estatiscamente ao modelo

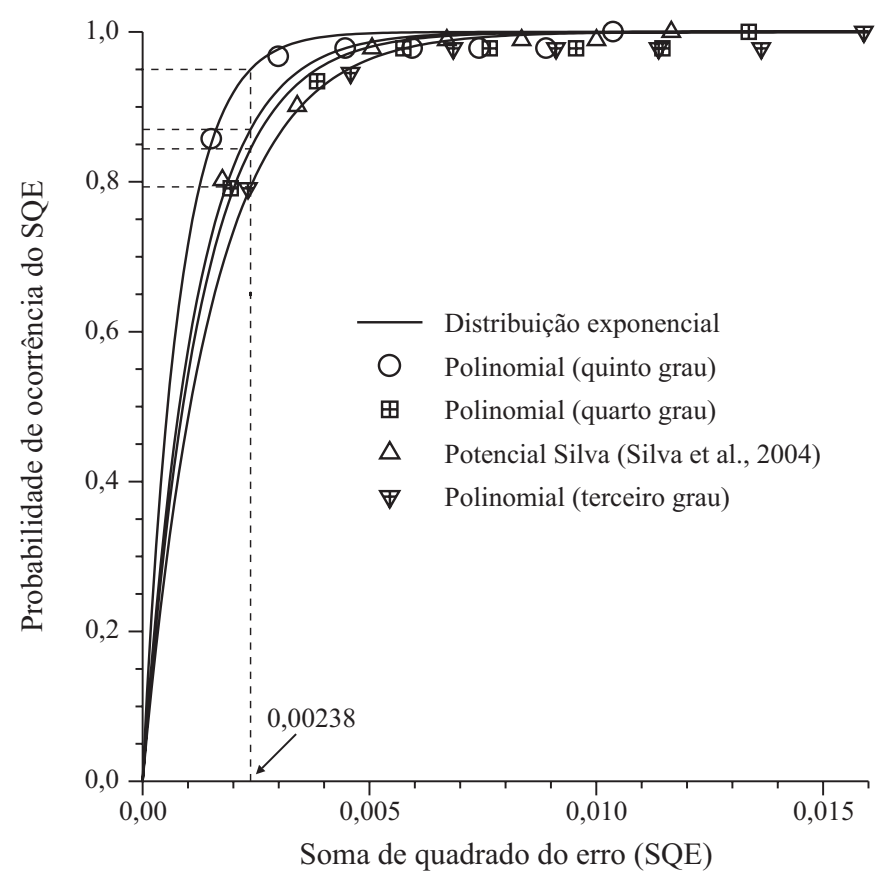

Figura 1. Distribuição estatística exponencial, ajustada às freqüências acumuladas dos valores de SQE de cada modelo com os respectivos níveis de probabilidade (segmentos pontilhados horizontais), correspondentes ao valor indicado de SQE igual a 0,00238

Potencial Silva, que apresentou um SQE médio de 0,00113. A função polinomial do terceiro grau, com um SQE médio de 0,00162 , foi a pior na comparação e a função polinomial do quarto grau, com um SQE médio de 0,00140, foi estatisticamente semelhante ao modelo Potencial Silva.

Tomando-se como referência a função polinomial do quinto grau e o valor específico de SQE igual a 0,00238 (Figura 1), correspondente ao nível de probabilidade de 95\%, o qual corresponde à chance de $5 \%$ ou menos, de se obter valores de SQE superiores a 0,00238, verificou-se que o modelo Potencial Silva apresentou 13,0\% de chance de proporcionar valores de SQE superiores a 0,00238 e as funções polinomiais do quarto e terceiro graus, a chance de 15,6 e 20,7\%, respectivamente; além disso, empregando-se a técnica de comparação de curvas (Silva \& Azevedo, 2002) com o teste $\mathrm{F}$ (Eq. 1) para determinar estatisticamente os níveis de significância na discriminação entre os modelos testados e se considerando o nível crítico de probabilidade de $5 \%$ da distribuição F (F = 4,75; $\mathrm{GL}_{\text {num }}=1$ e $\left.\mathrm{GL}_{\mathrm{den}}=12\right)$, verificouse, na comparação com o modelo Potencial Silva, que a função polinomial do terceiro grau, que apresentou o pior desempenho no ajuste aos dados medidos, resultou em um $\mathrm{F}=11,15$; a do quarto grau, que foi estatisticamente semelhante, apresentou um $\mathrm{F}=1,40$, e a do quinto grau, que foi superior ao modelo Silva, teve um $\mathrm{F}=19,14$ justificando, assim, sua proposição neste trabalho.

Para o desenvolvimento das expressões matemáticas que deverão ser usadas na análise de desempenho de sistemas de irrigação, com base na função polinomial do quinto grau adotaram-se, como exemplo de aplicação os dados da avaliação de desempenho de um sistema de irrigação por aspersão convencional (Frizzone \& Dourado Neto, 2003), cujos 
valores pontuais da coleta de água estão representados em forma de lâminas decrescentes, distribuídos em função da fração da área total irrigada (Figura 2).

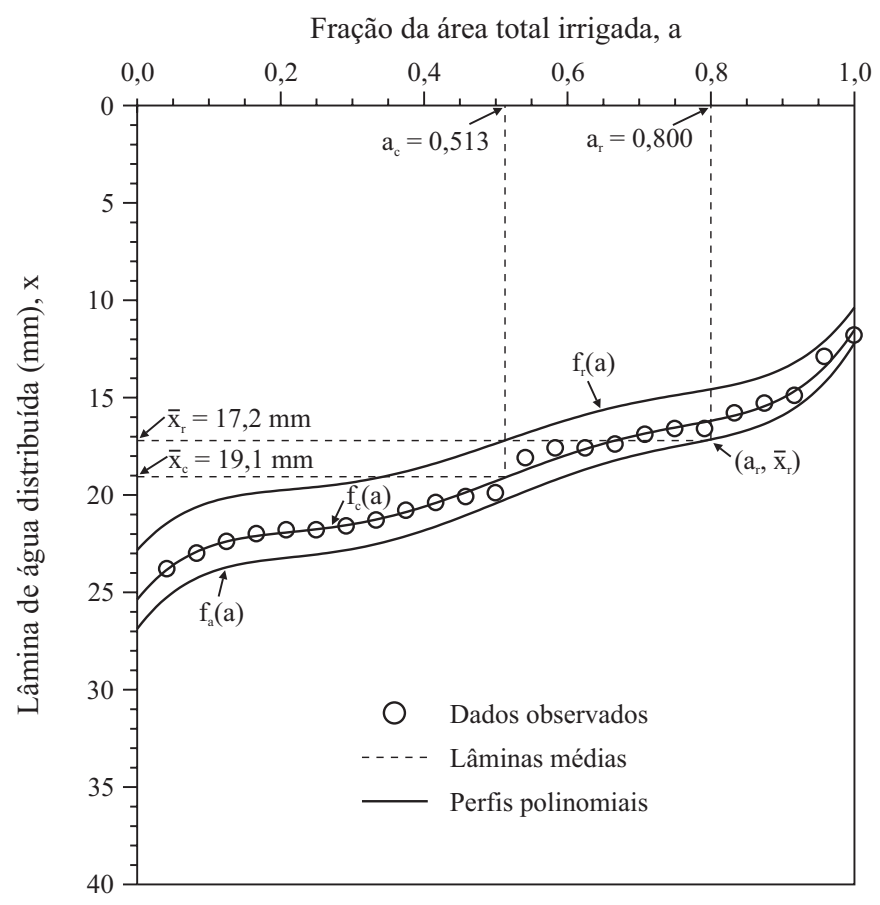

Figura 2. Ajuste do modelo polinomial do quinto grau utilizando os dados da avaliação de desempenho de um sistema de irrigação por aspersão convencional

Neste gráfico (Figura 2) estão representados os perfis de distribuição das lâminas de água coletadas, $\mathrm{f}_{\mathrm{c}}(\mathrm{a})$, na avaliação de desempenho da irrigação; as lâminas de água requeridas, $\mathrm{f}_{\mathrm{r}}(\mathrm{a})$, correspondente à demanda líquida do cultivo em uma irrigação e as lâminas de água a serem aplicadas, $\mathrm{f}_{\mathrm{a}}(\mathrm{a})$, para suprir a demanda do cultivo mais as perdas por percolação profunda em uma irrigação. Os valores correspondentes a $\bar{X}_{\mathrm{r}}$ e $\overline{\mathrm{X}}_{\mathrm{c}}$ representam, respectivamente, a lâmina média de água requerida pelo cultivo e a lâmina média coletada na avaliação; além disso, estão indicados também os valores equivalentes às áreas consideradas adequadamente irrigadas $\mathrm{a}_{\mathrm{c}}$ e $\mathrm{a}_{\mathrm{r}}$ associados aos perfis de distribuição de água $\mathrm{f}_{\mathrm{c}}(\mathrm{a})$ e $\mathrm{f}_{\mathrm{a}}(\mathrm{a})$, respectivamente.

Para o desenvolvimento das expressões matemáticas requeridas na determinação dos principais parâmetros de desempenho da irrigação, foram consideradas as seguintes definições e formulações (Tabela 1).

Para aplicar essa modelagem, o primeiro passo consistiu em se calcular a média da água coletada $\overline{\mathrm{X}}_{\mathrm{c}}$, integrando $\mathrm{f}_{\mathrm{c}}(\mathrm{a})$ no intervalo de 0 a 1 , seguindo a formulação indicada no item 2 da Tabela 1 . O passo seguinte foi estabelecer a igualdade $\mathrm{f}_{\mathrm{c}}\left(\mathrm{a}_{\mathrm{c}}\right)=\overline{\mathrm{x}}_{\mathrm{c}}$ (Figura 2) e determinar o valor de $\mathrm{a}_{\mathrm{c}}$ correspondente, por meio da técnica numérica de NewtonRapson, aplicada à formulação indicada no item 1 da Tabela 1. Uma vez conhecido o resultado de $\mathrm{a}_{c}$, os valores de CUC e CUD foram determinados aplicando-se as respectivas formulações indicadas nos itens 10 e 11, da Tabela 1; além disso, partindo-se do pressuposto de que, em geral, as lâminas médias da água requerida pelo cultivo nos vários eventos de irrigação não são necessariamente iguais à média da água coletada durante a avaliação de desempenho do sistema estabeleceu-se, como passo seguinte, a determinação do fator $\mathrm{S}$ (item 5 da Tabela 1), que transforma o perfil original $\mathrm{f}_{\mathrm{c}}(\mathrm{a})$ em $\mathrm{f}_{\mathrm{r}}(\mathrm{a})$, dividindo-se a lâmina média da água requerida em uma irrigação $\overline{\mathrm{x}}_{\mathrm{r}}$ pela média da água coletada durante a avaliação de desempenho $\overline{\mathrm{x}}_{\mathrm{C}}$ (Figura 2); em seguida, após ter sido estabelecido o valor de $\mathrm{a}_{\mathrm{r}}$, correspondente à meta, em termos da fração da área total irrigada que se deseja manter recebendo água em quantidades iguais ou superiores ao requerimento hídrico do cultivo, determinou-se o valor do fator R, denominado fator de adequação da irrigação em Silva et al. (2004), a partir da relação $f_{a}(a) / f_{r}(a)$, reconhecendo-se o fato de que no ponto $\left(\mathrm{a}_{\mathrm{r}}, \overline{\mathrm{x}}_{\mathrm{r}}\right)$ são válidas as igualdades: $\mathrm{f}_{\mathrm{a}}(\mathrm{a})=\overline{\mathrm{x}}_{\mathrm{r}}$ e $\mathrm{f}_{\mathrm{r}}(\mathrm{a})=\mathrm{f}_{\mathrm{r}}\left(\mathrm{a}_{\mathrm{r}}\right)$, como indicado no item 8 da Tabela 1. Este tipo de relação foi, de certa maneira, delineado em Hart \& Reynolds (1965) ao definirem seu fator de disponibilidade de água $\mathrm{F}_{\mathrm{a}}$ como sendo a relação entre a quantidade de água disponível e a quantidade total de água requerida pelo cultivo; assim sendo e uma vez conhecidos os valores de $\mathrm{R}, \mathrm{S}, \mathrm{a}_{\mathrm{r}}$ e $\overline{\mathrm{x}}_{\mathrm{r}}$, os demais indicadores de desempenho, relacionados com a eficiência de irrigação $\left(V_{E}, V_{D}, V_{U}, V_{A}, E_{A}\right)$, conforme se encontram definidos nos itens $12-16$ da Tabela 1 , puderam ser derivados do perfil de distribuição.

Para exemplificar a aplicação desse modelo se utilizaram os dados apresentados na Figura 2. O primeiro passo consistiu em determinar os parâmetros da polinomial $\mathrm{f}_{\mathrm{c}}(\mathrm{a})$, usando-se a técnica dos mínimos quadrados, resultando nos seguintes valores: $\mathrm{A}=25,3692 ; \mathrm{B}=-46,4821 ; \mathrm{C}=253,4448$; $\mathrm{D}=-670,4034 ; \mathrm{E}=753,4662 ; \mathrm{F}=-303,8731$. A etapa seguinte foi derivar a expressão matemática para o cálculo da média da água coletada $\overline{\mathrm{x}}_{\mathrm{c}}$, integrando-se a função $\mathrm{f}_{\mathrm{c}}(\mathrm{a})$ no intervalo de 0 a 1, resultando na Equação 2:

$$
\overline{\mathrm{x}}_{\mathrm{c}}=\mathrm{A}+\frac{\mathrm{B}}{2}+\frac{\mathrm{C}}{3}+\frac{\mathrm{D}}{4}+\frac{\mathrm{E}}{5}+\frac{\mathrm{F}}{6}
$$

a qual, aplicada ao caso exemplificado (Figura 2), resultou em $\bar{x}_{\mathrm{C}}=19,1 \mathrm{~mm}$. Empregando-se a técnica numérica iterativa de Newton-Rapson para determinação do valor de $\mathrm{a}_{\mathrm{c}}$, correspondente ao perfil original $f_{c}(a)$, com base na igualdade $\mathrm{f}_{\mathrm{c}}(\mathrm{a})=\overline{\mathrm{x}}_{\mathrm{c}}$ no ponto $\mathrm{a}=\mathrm{a}_{\mathrm{c}}$, obteve-se $\mathrm{a}_{\mathrm{c}}=0,513$, ou seja, caso as irrigações fossem feitas de acordo com o perfil de distribuição $\mathrm{f}_{\mathrm{c}}(\mathrm{a}), 51,3 \%$ da área irrigada estariam, recebendo lâminas de água em quantidades iguais ou superiores à média coletada, verificando-se através dos dados tabulados da distribuição de freqüências das lâminas de irrigação desse exemplo (Frizzone \& Dourado Neto, 2003) que o grau de adequação correspondente à lâmina média de 18,8 mm estaria, na realidade, em torno de $56,8 \%$, resultado este próximo ao valor aqui determinado. De qualquer sorte, as diferenças observadas, seja em termos de média como do grau de adequação, entre os resultados obtidos por Frizzone \& Dourado Neto (2003) e os determinados com a aplicação da função polinomial proposta, refletem principalmente a escolha inadequada deles do modelo de distribuição Normal para 
Tabela 1. Definições e formulações utilizadas na proposição do modelo polinomial do quinto grau para avaliação de desempenho de sistemas de irrigação

\begin{tabular}{|c|c|c|}
\hline Item & Descrição & Formulação $0^{(1)}$ \\
\hline 1. & $\begin{array}{l}\text { Modelo polinomial do quinto grau para representar o perfil de distribuição das lâminas de água coletada em } \\
\text { relação a fração da área total irrigada (a). }\end{array}$ & $\mathrm{f}_{\mathrm{c}}(\mathrm{a})=\mathrm{A}+\mathrm{Ba}+\mathrm{Ca}^{2}+\mathrm{Da}_{3}+\mathrm{Ea}^{4}+\mathrm{Fa}^{5}$ \\
\hline 2. & $\begin{array}{l}\text { Lâmina média de água coletada em uma avaliação de desempenho da irrigação, baseada no perfil de distri- } \\
\text { buição } f_{c}(\text { a). }\end{array}$ & $\overline{\mathrm{x}}_{\mathrm{c}}=\int_{0}^{1} \mathrm{f}_{\mathrm{c}}(\mathrm{a}) \mathrm{da}$ \\
\hline 3. & $\begin{array}{l}\text { Fração da área total irrigada com lâminas iguais ou superiores à média de água coletada na avaliação, } \\
\text { denominada grau de adequação, calculada segundo } \mathrm{f}_{\mathrm{c}}(\mathrm{a}) \text {. }\end{array}$ & $a_{c}=f_{c}^{-1}\left(\bar{x}_{c}\right) \quad$ (inverso de $f_{c}(a)$ avaliado em $\left.\bar{x}_{c}\right)$ \\
\hline 4. & Lâmina média de água requerida em uma irrigação, calculada em função da demanda hídrica do cultivo. & $\overline{\mathrm{X}}_{\mathrm{r}} \quad$ (dado de entrada) \\
\hline 5. & $\begin{array}{l}\text { Relação entre as lâminas médias de água coletada e requerida ou entre as lâminas de água dos perfis de } \\
\text { distribuição } f_{r}(a) \text { e } f_{c}(a) \text {. }\end{array}$ & $\mathrm{S}=\frac{\overline{\mathrm{X}}_{\mathrm{r}}}{\overline{\mathrm{X}}_{\mathrm{c}}}=\frac{\mathrm{f}_{\mathrm{r}}(\mathrm{a})}{\mathrm{f}_{\mathrm{c}}(\mathrm{a})}$ \\
\hline 6. & $\begin{array}{l}\text { Modelo polinomial do quinto grau para representar a distribuição da água requerida f } \mathrm{f}_{\text {( }}(\mathrm{a}) \text { em relação à fra- } \\
\text { ção da área total irrigada, cuja média é igual à demanda hídrica do cultivo em uma irrigaçâo. }\end{array}$ & $\mathrm{f}_{\mathrm{r}}(\mathrm{a})=\mathrm{S} \times \mathrm{f}_{\mathrm{c}}(\mathrm{a})$ \\
\hline 7. & Fração da área total estabelecida como a meta de área adequadamente irrigada a ser atingida. & $a_{r} \quad$ (dado de entrada) \\
\hline 8. & $\begin{array}{l}\text { Relação entre as lâminas de água das distribuições } \mathrm{f}_{\mathrm{a}}(\mathrm{a}) \text { e } \mathrm{f}_{\mathrm{r}}(\mathrm{a}) \text { ou entre a lâmina média requerida } \overline{\mathrm{x}}_{\mathrm{r}} \text { e a } \\
\text { lâmina específica calculada em } \mathrm{a}_{\mathrm{r}} \text { com o perfil de distribuiçâo } \mathrm{f}_{\mathrm{r}}(\mathrm{a})\end{array}$ & $R=\frac{f_{a}(a)}{f_{r}(a)}=\frac{\bar{x}_{r}}{f_{r}\left(a_{r}\right)}$ \\
\hline 9. & $\begin{array}{l}\text { Modelo polinomial do quinto grau para representar a distribuição de água aplicada em função da meta } \\
\text { estabelecida } \mathrm{a}_{\mathrm{r}} \text {, cujo traçado passa, necessariamente, pelo ponto }\left(\mathrm{a}_{\mathrm{r}}, \overline{\mathrm{X}}_{\mathrm{r}}\right) \text {. }\end{array}$ & $\mathrm{f}_{\mathrm{a}}(\mathrm{a})=\mathrm{R} \times \mathrm{f}_{\mathrm{r}}(\mathrm{a})=\mathrm{R} \times \mathrm{S} \times \mathrm{f}_{\mathrm{c}}(\mathrm{a})$ \\
\hline 10. & $\begin{array}{l}\text { Coeficiente de Uniformidade de Christiansen, equivalente à razão entre a média dos desvios da distribuição } \\
\mathrm{f}_{c}(\mathrm{a}) \text { e a média aplicada } \overline{\mathrm{x}}_{\mathrm{c}} \text {. }\end{array}$ & CUC $=1-\frac{1}{\bar{x}_{\mathrm{c}}}\left[\int_{0}^{\mathrm{a}_{\mathrm{f}}}\left[\mathrm{f}_{\mathrm{c}}(\mathrm{a})-\overline{\mathrm{x}}_{\mathrm{c}}\right] \mathrm{da}+\int_{\mathrm{a}_{\mathrm{c}}}^{1}\left[\overline{\mathrm{x}}_{\mathrm{c}}-\mathrm{f}_{\mathrm{c}}(\mathrm{a})\right] \mathrm{da}\right.$ \\
\hline 11. & $\begin{array}{l}\text { Coeficiente de Uniformidade de Distribuição, equivalente à razão entre a média do quartil inferior e a média } \\
\text { da distribuição } \mathrm{f}_{\mathrm{c}}(\mathrm{a}) \text {. }\end{array}$ & CUD $=\frac{4}{\bar{x}_{c}} \int_{3 / 4}^{1} f_{c}(a) d a$ \\
\hline 12. & $\begin{array}{l}\text { Volume de água aplicada em excesso, por unidade de área, calculado em relação à média de água requeri- } \\
\text { da } \bar{x}_{t} \text {, baseado na distribuição } f_{a}(a) \text {. }\end{array}$ & $\mathrm{V}_{\mathrm{E}}=\int_{0}^{\mathrm{a}}\left[\mathrm{R} \times \mathrm{S} \times \mathrm{f}_{\mathrm{c}}(\mathrm{a})-\overline{\mathrm{x}}_{\mathrm{t}}\right] \mathrm{da}$ \\
\hline 13. & $\begin{array}{l}\text { Volume de água aplicada em deficiência, por unidade de área, calculado em relação à média de água reque- } \\
\text { rida } \bar{x}_{t} \text {, baseado na distribuiçẫo } f_{a}(a) \text {. }\end{array}$ & $\mathrm{V}_{\mathrm{D}}=\int_{\mathrm{a}_{\mathrm{r}}}^{1}\left[\overline{\mathrm{x}}_{\mathrm{r}}-\mathrm{R} \times \mathrm{S} \times \mathrm{f}_{\mathrm{c}}(\mathrm{a})\right] \mathrm{da}$ \\
\hline 14. & $\begin{array}{l}\text { Volume útil, por unidade de área, calculado em relação à média de água requerida, correspondente às } \\
\text { lâminas de água iguais ou inferiores } a \bar{x}_{r} \text { em relação à distribuição } f_{a}(a) \text {. }\end{array}$ & $\mathrm{V}_{\mathrm{D}}=\overline{\mathrm{x}}_{\mathrm{r}} \mathrm{a}_{\mathrm{r}}+\int_{\mathrm{a}_{\mathrm{r}}}^{1}\left[\mathrm{R} \times \mathrm{S} \times \mathrm{f}_{\mathrm{c}}(\mathrm{a})\right] \mathrm{da}$ \\
\hline 15. & Volume de água aplicada, por unidade de área, baseado na distribuição $\mathrm{f}_{\mathrm{a}}(\mathrm{a})$. & $\mathrm{V}_{\mathrm{A}}=\int_{0}\left[\mathrm{R} \times \mathrm{S} \times \mathrm{f}_{\mathrm{c}}(\mathrm{a})\right] \mathrm{da}$ \\
\hline 16. & $\begin{array}{l}\text { Eficiência de aplicação de água (\%), baseada na distribuição } \mathrm{f}_{\mathrm{a}}(\mathrm{a}) \text { e se considerando a lâmina média reque- } \\
\text { rida } \bar{x}_{\mathrm{r}} \text { como a água útil. }\end{array}$ & $\mathrm{E}_{\mathrm{A}}=100 \frac{\mathrm{V}_{\mathrm{U}}}{\mathrm{V}_{\mathrm{A}}}$ \\
\hline
\end{tabular}

(1) $A, B, C, D, E$ e $F$ são parâmetros da função polinomial do quinto grau e os valores de $a_{c}$ e $a_{r}$ são restritos ao intervalo de 0 a 1

representar os valores de água coletados e calcular os parâmetros da análise de desempenho dessa irrigação; tal conclusão está bem fundamentada em Silva et al. (2006) que demonstraram ser o modelo Normal o pior dentre os modelos não-lineares testados, o que torna questionável a utilização generalizada deste modelo para representar perfis de distribuição da água aplicada pela irrigação.

Em relação ao cálculo dos indicadores de uniformidade da irrigação, ressalta-se que as informações requeridas na derivação das expressões matemáticas para o cálculo do CUC e CUD (itens 10 e 11 da Tabela 1) estão inteiramente contidas no perfil de distribuição da água coletada (Figura 2). As equações desenvolvidas foram as seguintes:

$$
\begin{aligned}
& \text { CUC }=\left[1-\frac{2 a_{c}}{\bar{x}_{c}}\left(A+\frac{B}{2} a_{c}+\frac{C}{3} a_{c}^{2}+\frac{D_{3}}{4} a_{c}^{3}+\frac{E}{5} a_{c}^{4}+\frac{F}{6} a_{c}^{5}-\bar{x}_{c}\right)\right] \\
& C U D=\frac{4}{\bar{x}_{c}}\left[\bar{x}_{c}-A\left(\frac{3}{4}\right)-\frac{B}{2}\left(\frac{3}{4}\right)^{2}-\frac{C}{3}\left(\frac{3}{4}\right)^{3}-\frac{D}{4}\left(\frac{3}{4}\right)^{4}-\frac{E}{5}\left(\frac{3}{4}\right)^{5}-\frac{F}{6}\left(\frac{3}{4}\right)^{6}\right]
\end{aligned}
$$

Tendo em vista os dados da avaliação de desempenho (Figura 2) e se aplicando as respectivas equações 3 e 4, obtiveram-se os valores de CUC $=85,8 \%$ e CUD $=78,6 \%$. Com esses mesmos dados, Frizzone \& Dourado Neto (2003) encontraram os seguintes valores: CUC $=85,1 \% \mathrm{e}$ CUD $=77,7 \%$, os quais são bastante similares aos calculados com a representação das lâminas coletadas pela função polinomial do quinto grau.

$\mathrm{Na}$ derivação das expressões matemáticas relacionadas com a eficiência de irrigação, considerou-se o fato de que o requerimento hídrico do cultivo varia ao longo de seu ciclo e, por isso, o perfil de distribuição da água coletada $\mathrm{f}_{\mathrm{c}}(\mathrm{a})$ na avaliação do sistema de irrigação raramente coincide com o perfil de distribuição da água requerida $\mathrm{f}_{\mathrm{r}}(\mathrm{a})$. Assumindo a proporcionalidade entre as lâminas de água desses dois perfis utilizou-se, então, o fator $\mathrm{S}=\overline{\mathrm{X}}_{\mathrm{r}} / \overline{\mathrm{X}}_{\mathrm{c}}$ e a relação $\mathrm{f}_{\mathrm{r}}(\mathrm{a})=\mathrm{Sf}_{\mathrm{c}}(\mathrm{a})$ (tens 5 e 6 da Tabela 1) para estimar $\mathrm{f}_{\mathrm{r}}(\mathrm{a})$. Para exemplificar a aplicação deste procedimento se assumiu, para dada irrigação, uma lâmina média requerida de 17,2 mm. Com base 
neste valor se calculou $S=0,90(=17,2 / 19,1)$ para então gerar o perfil correspondente da distribuição de água requerida $\mathrm{f}_{\mathrm{r}}(\mathrm{a})$ representado na Figura 2.

Por outro lado, o perfil da água aplicada $\mathrm{f}_{\mathrm{a}}(\mathrm{a})$, em dada irrigação depende do grau de adequação $\mathrm{a}_{\mathrm{r}}$ pretendido, caso em que, partindo-se da premissa estabelecida de que a lâmina média de água requerida $\overline{\mathrm{x}}_{\mathrm{r}}$ deve cruzar com o perfil $\mathrm{f}_{\mathrm{a}}(\mathrm{a})$ no ponto $\left(a_{r}, \bar{x}_{r}\right)$, então o valor de $R=f_{a}(a) / f_{r}(a)$ pôde ser determinado pela relação $\mathrm{R}=\overline{\mathrm{x}}_{\mathrm{r}} / \mathrm{f}_{\mathrm{r}}\left(\mathrm{a}_{\mathrm{r}}\right)$ (item 8 da Tabela 1 ). Para $\mathrm{o}$ caso específico desse exemplo (Figura 2), o valor de $\mathrm{R}$ foi de 1,18 para um grau de adequação de $80 \%$, significando que seria necessário realizar uma majoração de $18 \%$ na lâmina média requerida para se alcançar a meta de $80 \%$ da área, recebendo lâminas de água aplicada superiores à média requerida. Caso o grau de adequação, para este exemplo, fosse ampliado para $90 \%$, o valor de R seria de 1,27 , ou seja, a lâmina média requerida deveria ser majorada em $27 \%$ o que, certamente, aumentaria as perdas por percolação profunda e reduziria a eficiência de aplicação de água da irrigação.

Tomando-se por base o perfil de distribuição da água coletada $\mathrm{f}_{\mathrm{c}}(\mathrm{a})$ e os valores conhecidos de $\mathrm{R}, \mathrm{S}, \mathrm{a}_{\mathrm{r}}$ e $\overline{\mathrm{x}}_{\mathrm{r}}$, concluise que as expressões matemáticas dos demais indicadores de desempenho, relacionados com a eficiência de irrigação $\left(V_{E}\right.$, $\mathrm{V}_{\mathrm{D}}, \mathrm{V}_{\mathrm{U}}, \mathrm{V}_{\mathrm{A}}, \mathrm{E}_{\mathrm{A}}$ ) foram derivadas utilizando-se suas respectivas formulações (itens 12-16 da Tabela 1), o que resultou nas seguintes equações:

$$
\begin{aligned}
& \mathrm{V}_{\mathrm{E}}=\operatorname{RS}\left[A \mathrm{a}_{\mathrm{r}}+\frac{\mathrm{B}}{2} \mathrm{a}_{\mathrm{r}}^{2}+\frac{\mathrm{C}}{3} \mathrm{a}_{\mathrm{r}}^{3}+\frac{\mathrm{D}}{4} \mathrm{a}_{\mathrm{r}}^{4}+\frac{\mathrm{E}}{5} \mathrm{a}_{\mathrm{r}}^{5}+\frac{\mathrm{F}}{6} \mathrm{a}_{\mathrm{r}}^{6}\right]-\overline{\mathrm{x}}_{\mathrm{r}} \mathrm{a}_{\mathrm{r}} \\
& \mathrm{V}_{\mathrm{D}}=\overline{\mathrm{x}}_{\mathrm{r}}\left(1-\mathrm{a}_{\mathrm{r}}\right)- \\
& \operatorname{RS} S\left[\mathrm{~A}\left(1-\mathrm{a}_{\mathrm{r}}\right)+\frac{\mathrm{B}}{2}\left(1-\mathrm{a}_{\mathrm{r}}^{2}\right)+\frac{\mathrm{C}}{3}\left(1-\mathrm{a}_{\mathrm{r}}^{3}\right)+\frac{\mathrm{D}}{4}\left(1-\mathrm{a}_{\mathrm{r}}^{4}\right)+\frac{\mathrm{E}}{5}\left(1-\mathrm{a}_{\mathrm{r}}^{5}\right)+\frac{\mathrm{F}}{6}\left(1-\mathrm{a}_{\mathrm{r}}^{6}\right)\right]
\end{aligned}
$$$$
\mathrm{V}_{\mathrm{U}}=\overline{\mathrm{X}}_{\mathrm{r}} \mathrm{a}_{\mathrm{r}}+
$$$$
\operatorname{RS}\left[A\left(1-a_{r}\right)+\frac{B}{2}\left(1-a_{r}^{2}\right)+\frac{C}{3}\left(1-a_{r}^{3}\right)+\frac{D}{4}\left(1-a_{r}^{4}\right)+\frac{E}{5}\left(1-a_{r}^{5}\right)+\frac{F}{6}\left(1-a_{r}^{6}\right)\right](7)
$$$$
\mathrm{V}_{\mathrm{A}}=\mathrm{RS}\left[\mathrm{A}+\frac{\mathrm{B}}{2}+\frac{\mathrm{C}}{3}+\frac{\mathrm{D}}{4}+\frac{\mathrm{E}}{5}+\frac{\mathrm{F}}{6}\right]=\mathrm{R} \mathrm{S} \overline{\mathrm{x}}_{\mathrm{c}}
$$

$\mathrm{E}_{\mathrm{A}}=100 \frac{\mathrm{V}_{\mathrm{U}}}{\mathrm{V}_{\mathrm{A}}}$

Com o mesmo exemplo (Figura 2), e os valores de $\mathrm{a}_{\mathrm{r}}=0,80, \overline{\mathrm{x}}_{\mathrm{r}}=17,2 \mathrm{~mm}$ e $\mathrm{R}=1,18$, os volumes de água por unidade de área, e a eficiência de aplicação dessa irrigação, foram os seguintes: $\mathrm{V}_{\mathrm{E}}=3,4 \mathrm{~mm}, \mathrm{~V}_{\mathrm{D}}=0,3 \mathrm{~mm}$, $\mathrm{V}_{\mathrm{U}}=16,9 \mathrm{~mm}, \mathrm{~V}_{\mathrm{A}}=20,3 \mathrm{~mm}$ e $\mathrm{E}_{\mathrm{A}}=83,3 \%$. Caso o grau de adequação fosse elevado para 90\%, mantendo o mesmo valor da lâmina média pressuposta como requerida de $\overline{\mathrm{X}}_{\mathrm{r}}=17,2 \mathrm{~mm}$, os resultados correspondentes desta análise de desempenho seriam os seguintes: $\mathrm{R}=1,27, \mathrm{~V}_{\mathrm{E}}=4,8 \mathrm{~mm}$, $\mathrm{V}_{\mathrm{D}}=0,2 \mathrm{~mm}, \mathrm{~V}_{\mathrm{U}}=17,0 \mathrm{~mm}, \mathrm{~V}_{\mathrm{A}}=21,8 \mathrm{~mm}$ e $\mathrm{E}_{\mathrm{A}}=78,0 \%$. Esses valores indicam que haveria um aumento de 41,2\% na água aplicada em excesso; 7,4\% na água total aplicada e $0,6 \%$ no volume útil aplicado e um decréscimo de 33,3\% no volume de água aplicada em deficiência e uma redução de 5,3\% na eficiência de aplicação.
Ressalta-se, ainda, que nessa conceituação (Silva et al., 2004), a majoração da lâmina média de água requerida $\overline{\mathrm{x}}_{\mathrm{r}}$ ou lâmina líquida de irrigação, se baseia somente na aplicação do fator $\mathrm{R}$ e que a eficiência de aplicação $\mathrm{E}_{\mathrm{A}}$ e os demais parâmetros correlatos são resultados dessa majoração; neste aspecto, deve-se destacar que a prática geral adotada para transformar a lâmina líquida de irrigação em lâmina bruta tem sido bastante variada. Por exemplo, Andrade Júnior et al. (2001) utilizaram a eficiência de aplicação $\left(E_{A}\right)$; Hamada \& Testezlaf (1995), o coeficiente de uniformidade de distribuição (CUD); Paz et al. (1997), o coeficiente de uniformidade de Christiansen (CUC). Na realidade, as alternativas baseadas tanto no CUC como no CUD apresentam o viés de não considerarem variável a meta pretendida de área adequadamente irrigada $\mathrm{a}_{\mathrm{r}}$ e a alternativa baseada na $\mathrm{E}_{\mathrm{A}}$ só seria estritamente verdadeira para $a_{r}=1$, condição em que $\mathrm{E}_{\mathrm{A}}$ seria igual ao inverso de $\mathrm{R}$.

Considerando-se, ainda, a avaliação de desempenho exemplificada (Figura 2), caso fosse utilizado o indicador CUC $=85,8 \%$ para majorar a lâmina líquida, haveria uma aplicação de água que corresponderia a uma majoração de $16,6 \%$ do requerimento hídrico, enquanto se fosse utilizado o $C U D=78,6 \%$, essa majoração seria de 27,2\%. Ambos os casos resultariam, obviamente, em eficiências distintas de aplicação de água e diferentes daquela calculada com a aplicação do fator R aqui indicado. Finalmente, ressalta-se que, com a aplicação deste fator, os três principais indicadores da avaliação de desempenho da irrigação (uniformidade, eficiência e grau de adequação) ficam integrados em um só parâmetro simplificando, assim, a interpretação dos resultados.

Na aplicação desses procedimentos de correção do perfil de distribuição da água coletada, realizando-se seu deslocamento para cima ou para baixo, dependendo da lâmina média requerida e do grau de adequação estabelecido, parte-se do pressuposto de que o formato do perfil de distribuição da água coletada na avaliação de desempenho do sistema de irrigação se manterá inalterado, independente do tempo de aplicação de água, no caso de sistemas de irrigação estacionários ou da velocidade de deslocamento do equipamento de distribuição de água, no caso de sistemas de irrigação móveis. Este pressuposto poderá não ser necessariamente verdadeiro, pois vários fatores intervenientes, decorrentes de variações hidráulicas, climáticas e topográficas, entre outros, podem afetar o padrão de distribuição de água do sistema de irrigação (Frizzone \& Dourado Neto, 2003) e, conseqüentemente, alterar o formato do perfil de distribuição da água aplicada em uma irrigação, razão por que se recomenda que os sistemas de irrigação sejam avaliados nas condições de operação mais próximas possíveis daquelas em que o sistema irá funcionar na maioria das vezes.

\section{CONCLUSÕES}

1. O modelo polinomial do quinto grau é o recomendado para representar os perfis de distribuição de água aplicada em sistemas de irrigação. 
2. As relações matemáticas desenvolvidas para o cálculo dos principais indicadores de uniformidade e dos parâmetros de eficiência de aplicação de água facilitam a análise do desempenho e simplificam sua utilização em estratégias de manejo da água da irrigação.

3. O fator R é o parâmetro de manejo da irrigação que deve ser usado para transformar a lâmina média de água requerida pelo cultivo em lâmina bruta e, assim, integrar, em um só termo, os indicadores de uniformidade, eficiência e grau de adequação da avaliação de desempenho da irrigação.

\section{AgRAdECIMENTOS}

À FINEP/CT-HIDRO, pelo apoio financeiro ao projeto RAIOBA; ao pesquisador da Embrapa Cerrados, Lineu Neiva Rodrigues, e a Ana Isabel dos Santos Grenho, pelo levantamento e cessão de parte da base de dados utilizada neste estudo.

\section{LITERATURA CITADA}

Andrade Júnior, Frizzone, J. A.; Bastos, E. A.; Cardoso, M. J.; Rodrigues, B. H. N. Estratégias ótimas de irrigação para a cultura da melancia. Pesquisa Agropecuária Brasileira, v.36, n.2, p.301-305, 2001.

Chaudry, F. H. Nonuniform sprinkler irrigation application efficiency. Journal of the Irrigation and Drainage Division, v.204, n.2, p.165-178, 1978.

Draper, N.; Smith, H. Applied regression analysis. 3.ed. New York: John Wiley \& Sons, 1998. 706p.

Elliot, R. L.; Nelson, J. D.; Lofts, J. C.; Hart, W. E. Comparison of sprinkler uniformity models. Journal of Irrigation and Drainage Division, v.106, n.4, p.321-330, 1980.
Frizzone, J. A.; Dourado Neto, D. D. Avaliação de sistema de irrigação. In: Miranda, J. H.; Pires, R. C. de M. (ed.). Irrigação. Piracicaba: FUNEP, 2003. p.573-651.

Hamada, E.; Testezlaf, R. Desenvolvimento e produtividade da alface submetida a diferentes lâminas de água através da irrigação por gotejamento. Pesquisa Agropecuária Brasileira, v.30, n.9, p.1201-1209, 1995.

Hart, W. E.; Reynolds, W. N. Analytical design of sprinkler systems. Transactions of the American Society of Agricultural Engineers, v.8, n.1, p.83-85, 89, 1965.

Karmelli, D. Distribution pattern and losses for furrow irrigation. Journal of the Irrigation and Drainage Division, v.104, n.1, p.59-69, 1978.

Lima, J. E. F. W.; Silva, E. M. da; Carvalho, N. de O. Comparação de modelos matemáticos para o traçado de curvas granulométricas de sedimentos do leito de rios. Revista Brasileira de Recursos Hídricos, v.11, n.1, p.91-98, 2006.

Paz, V. P. da S.; Frizzone, J. A.; Botrel, T. A.; Folegatti, M. V. Redução da receita líquida por déficit ou excesso de água na cultura do feijoeiro. Pesquisa Agropecuária Brasileira, v.32, n.9, p.869-875, 1997.

Silva, E. M. da; Azevedo, J. A. de. Influência do período de centrifugação na curva de retenção de água em solos de Cerrado. Pesquisa Agropecuária Brasileira, v.37, n.10, p.14871494, 2002.

Silva, E. M. da; Lima, J. E. F. W.; Azevedo, J. A. de; Rodrigues, L. N. Proposição de um modelo matemático para a avaliação do desempenho de sistemas de irrigação. Pesquisa Agropecuária Brasileira, v.39, n.8, p.741-748, 2004.

Silva, E. M. da; Lima, J. E. F. W.; Azevedo, J. A. de; Rodrigues, L. N. Valores de tensão na determinação da curva de retenção de água de solos do Cerrado. Pesquisa Agropecuária Brasileira, v.41, n.2, p.323-330, 2006.

Souza, G. da S. e. Introdução aos modelos de regressão linear e nãolinear. Brasília: EMBRAPA-SPI/EMBRAPA-SEA, 1998. 505p. 